\title{
Enhancing the angular stability of Artificial Magnetic Conductors through lumped inductors
}

\author{
H. F. Álvarez ${ }^{\mathrm{a}}$, M. E. de $\operatorname{Cos}^{\mathrm{a}}$, S. García ${ }^{\mathrm{a}}$ and F. Las-Heras ${ }^{\mathrm{a}}$ \\ a Área de Teoría de la Señal y Comunicaciones. Dpt. Ingeniería Eléctrica, Universidad de Oviedo. \\ Edificio Polivalente, Mod. 8. E-33203 Gijón, Asturias, Spain \\ hfernandez@tsc.uniovi.es
}

\begin{abstract}
In this paper a novel useful method to efficiently improve the angular stability of AMCs is introduced. Aiming to obtain an AMC with fixed design specifications (resonance frequency, proper bandwidth, maximum size and specific dielectric), the method relies on increasing the grid inductance through the introduction of lumped inductors. To date, lumped capacitors and/or inductors have been used to decrease the unit-cell dimensions. However, to the author's knowledge their potential applications on angular stability enhancement have not been explored yet. The proposed method is applied to non-angular stable AMC comprising square loop-based unit-cells on a $\mathrm{RO4003C}$ dielectric. The validity of the method is demonstrated not only through electromagnetic simulations but also using an equivalent circuit model which clearly explains the behavior of the AMC. Moreover, some guidelines are given in order to choose a proper lumped inductance. At the end, comparison with previous literature contributions shows the great potential of this method.
\end{abstract}

Keywords -- AMC, angular stability, lumped inductors, miniaturization.

\section{Introduction}

Artificial magnetic conductors (AMC) have been widely studied over the last few years, mostly due to their applications on antennas. In fact, using them as a ground plane on antennas improves their radiation properties (efficiency and gain) and even their bandwidth [1]-[5]. Moreover, miniaturizing the antenna profiles can also be possible [6]. The radar cross section (RCS) reduction [7] is another potential application in fields like detection, surveillance and sensing of people and/or objects. In addition, the decreasing of the specific absorption rate (SAR) in "weareable" devices [5], [8] has a great impact in medical applications among others.

AMCs are resonant structures which can be considered two dimensional metamaterials and hence they are also classified as metasurfaces [9], [10]. The most common way to build up AMCs is through grounded metallo-dielectric frequency selective surfaces (FSS). Thus, it comprises unit-cells with a top metallic geometry on a grounded dielectric slab. An infinite plane wave faces a high impedance surface (HIS) when it impinges on the AMC, provided the wave frequency equals the AMC's resonance one. Consequently, this wave is reflected in phase [11]. This phenomenon can be easily explained if the transmission line theory is used to model the structure. Doing so, the reflection coefficient of the AMC illuminated by a normally incident plane wave can be represented as [12]:

$$
\Gamma(\mathrm{w}, \theta, \phi)=\frac{Z_{s}(\mathrm{w}, \theta, \phi)-\eta_{0}}{Z_{S}(\mathrm{w}, \theta, \phi)+\eta_{0}}
$$

where $\eta_{0}$ is the free space impedance and $Z_{s}(w, \theta, \phi)$ is the effective impedance of the structure. This impedance varies with the frequency $(w)$, polarization $(\phi)$ and incidence angle $(\theta)$ of the incident wave. Providing the periodicity of the structure is much smaller than the operational wavelength, this impedance can be represented as a parallel connection between the grid impedance and the metal-backed dielectric impedance (also known as grounded dielectric impedance) [13].

AMCs are commonly analyzed by computing the phase of the reflection coefficient on their surface. It varies with frequency between $\pm 180^{\circ}$ and crosses $0^{\circ}$ at the $\mathrm{AMC}$ 's resonance frequency. Therefore, only at a certain frequency the wave is reflected totally in phase. However, around this frequency the wave is reflected more in phase than out of phase. Thus, the AMC behavior is mostly considered to be within $\pm 90^{\circ}$ in the reflection coefficient phase [5],[11],[13]-[15]. Although this is the bandwidth used in most of the articles in the literature, some applications require more restricted considerations [16], [17].

A designer usually looks for a wide AMC's operation bandwidth over a large variety of polarizations and angles of incidence. Therefore, observing the expression of the reflection coefficient (1) and taking into consideration the previous remarks, it is obvious that the main limitations of AMCs are in the 
bandwidth and the angular stability. The bandwidth limitations have been studied in many articles using multiresonance structures [7],[8],[18]-[19]. Some solutions are to stack several layers [18] or using unitcells with close resonances [7]. However, not so many contributions deal with the angular stability of AMCs [1]-[4],[13],[20]-[22]. Therefore, this will be the aim of this paper.

When an AMC is used as a reflector of an electrically small antenna, the previous mentioned improvements in the antenna performance are reduced in the case of involving a non-angular stable AMC. Indeed, the different harmonics created by the antenna impinges on the AMC at different angles, so they are reflected with different phase values and hence the contributions will not always be added constructively (in phase) [1]-[4]. Therefore, the improvement in the radiation efficiency is marginal. In fact, in this case this improvement is more due to the suppression of surface waves than to the constructive interference contributions between the antenna and the AMC.

Theoretical basis for a uniaxial material which exhibits angular independence high impedance behavior over a wide range of frequencies are presented in [20]. However, it is not easily realizable in practice for whatever polarization of the incident wave.

One of the first works on angular stability of AMCs was carried out on [21]. It is known that when vertical vias are excited they give rise to electrical currents which are sometimes used by several authors to improve AMC's angular stability. However, these vias are just excited when the electric field is along them which only occurs under transverse magnetic (TM) polarized incident wave. Therefore, in [21] the duality principle is used and it is supposed that the same effect can be obtained for transverse electric (TE) polarization if vertical magnetic currents are excited. Thence, spiral elements are introduced to improve the angular stability. Another interesting article on angular stability is [4]. Several grids together with grounded dielectric slabs with and without vias are analyzed with the aim of finding the best combination of them that avoid angular dependence in the AMC. This best choice is the use of a seriesresonant grid (SRG) (grid can be modeled as a series connection of an inductance $\left(L_{g}\right)$ and a capacitance $\left(C_{g}\right)$ ) above a grounded dielectric slab without vias. Although analytically it seems to be no angular dependence, electromagnetic simulations show a small dependence. This is attributed by the authors of the article to the approximations that have been used to obtain the grid models. In [1] authors gather the work done in [4] and summarize the requirements that an AMC structure should fulfill in order to achieve angular independence. Such requirements are:

- The grid should be closely coupled (period $<<\lambda$ ) and consist of a SRG.

- The dielectric slab have not to contain vias and must be thin $\left(k_{d} h \ll 1\right)$, where $k_{d}=w \sqrt{\varepsilon_{0} \varepsilon_{r} \mu_{0}}$ and $h$ is the thickness of the dielectric.

- The period should be comparable with $h$. This is essential in order to use the expressions given in [4] to model the structure.

In [1], authors use a double layer Jerusalem cross. This arrangement increases the capacitance coupling between neighbor unit-cells. Therefore, the condition of closely coupled grid (first condition) is better satisfied and the periodicity of the unit-cell is compressed. Consequently, the AMC's angular stability is improved. In [2], a similar procedure to the one in [1] is employed. In this case the capacitance is increased due to both the shape of the unit-cell and the introduction of a double layer structure. However, in both articles double layer structures are used so the thickness and the fabrication complexity of the AMCs are increased. Moreover, the study of the angular stability is performed at only one frequency. An array of one dimensional periodic wires distributed in a non-uniform way is used in [3] in order to improve the angular dependency of the proposed AMC. A dipole antenna is used to corroborate that this non-uniformity distribution improves the angular stability of the structure and hence the performance of the antenna. Another study on the angular dependence of a perturbed array of dipoles is carried out in [23]. However, this structure only works for TE incident waves. A more detailed angular stability analysis was carried out in [24] for AMCs based on an array of hexagonal as well as square dipoles.

On the other hand, the introduction of lumped components has been studied with the aim of miniaturizing AMCs [25], but no angular stability analysis has been tackled.

Although some breakthroughs have been introduced in the realm of AMCs' angular stability, there is still much work to do on this topic. Thus, the aim of this paper is to introduce a new idea for improving the AMCs' angular stability. As it was mentioned some papers tend to increase the coupling between neighbor unit-cells (increase the grid capacitance) with the aim of improving the angular stability. However, to the authors' best knowledge there is no previous work analyzing the effect of altering the grid inductance. So this will be the focus of this contribution. Moreover, the advantages of tailoring the grid inductance are highlighted and compared with other contributions where the angular stability is pursued. 


\section{Description of the problem and initial designs}

As it was pointed out in the introduction, this work aims to improve the angular stability of AMCs and give some guidelines to achieve it. This angular stability depends on both the dielectric (thickness and permittivity) and the grid characteristics (unit-cell's metallization geometry and size (periodicity) as compared to the dielectric thickness) of the AMC. Once the dielectric substrate and the unit-cell periodicity are fixed both the angular stability and the AMC bandwidth only depend on the unit-cell's metallization geometry. It is well known that by increasing the substrate relative dielectric permittivity and/or reducing its thickness the angular stability is improved. However, whichever of these techniques gives rise to a shift in the resonance frequency and a narrowing in the AMC bandwidth. Therefore, a trade-off solution concerning these two parameters must be adopted [13].

In this work both the dielectric substrate and the AMC unit-cell periodicity will be fixed and hence, conclusions regarding the unit-cell's metallization geometry influence on the angular stability will be obtained.

From previous works, it has been concluded that loop-based unit-cells are more angularly stable than patch-based ones [13]. In addition, the hexagonal-shaped unit-cells outperform the square-shaped ones as regards angular stability provided a proper gap distance between neighbor unit-cells is considered. Since the aim of this work is to obtain an effective method for improving the AMCs' angular stability, a unitcell's metallization geometry from a non-angular stable AMC is chosen. Therefore, the square loop-based unit-cell (LUC) of Fig. 1(a) is considered as starting point. The geometry parameters of the LUC are as follows: $p=7.3 \mathrm{~mm}, w_{e}=6.3 \mathrm{~mm}$, and $w_{i}=3.4 \mathrm{~mm}$. The dielectric substrate is a RO4003C $\left(\varepsilon_{r}=3.38\right.$ and loss tangent 0.0027 ) with a dielectric thickness of $1.524 \mathrm{~mm}$.

The behavior of the LUC based AMC can be modeled by the equivalent circuit of the Fig. 1(b). The grid impedance in this case is a series connection of a grid inductance $\left(L_{g}\right)$ and a grid capacitance $\left(C_{g}\right)$ as it has been explained in [26]. The grounded dielectric slab can be modeled as an inductance $\left(L_{d}\right)$ provided that its thickness is electrically small with respect to the operational wavelength. Therefore, the equivalent (surface) impedance of the $\mathrm{AMC} Z_{s}(\mathrm{w}, \theta, \phi)$ can be expressed as

$$
Z_{s}(\mathrm{w}, \theta, \phi)=\frac{\mathrm{jwL} \mathrm{L}_{\mathrm{d}}\left(1-w^{2} L_{g} C_{g}\right)}{1-w^{2} C_{g}\left(L_{g}+L_{d}\right)}
$$

and the reflection coefficient can be calculated from (1). Analyzing the frequency variation of the latter one, two interesting frequencies, where the imaginary part of the reflection coefficient vanishes, appear:

$$
\begin{aligned}
& f_{1}=\frac{1}{2 \pi \sqrt{L_{g} C_{g}}} \\
& f_{2}=\frac{1}{2 \pi \sqrt{L_{g} C_{g}+L_{d} C_{g}}}
\end{aligned}
$$

The first one (3) brings about a zero in the equivalent (surface) impedance of the AMC and the latter one (4) a pole. Therefore, it is the frequency computed by (4) which causes the behavior of the structure as an AMC (in phase reflection).

Ansoft HFSS commercial software is used throughout this paper to numerically compute the AMCs' reflection coefficient phase. A single unit-cell can be used to characterize the behavior of the whole structure, provided both proper periodic boundary conditions are applied on the unit-cell and the excitation modes are defined using the Bloch-Floquet theory. Both modes, traverse electric (TE) and transverse magnetic (TM), are considered in the analysis. The electric field is normal to the incidence plane for TE modes whereas for TM modes the magnetic field is the normal one.

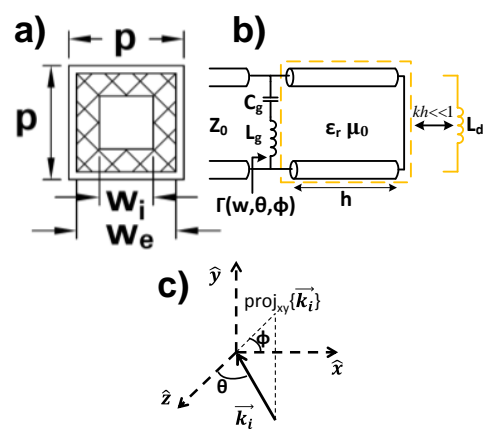

Fig. 1. (a) Geometry of LUC (top view). (b) Equivalent circuit model of LUC based AMC. (c) Coordinate system for angular stability analysis. 
The reflection coefficient phase of the LUC based AMC is simulated, varying the polarization angle $(\phi)$ from $0^{\circ}$ to $90^{\circ}$ in steps of $15^{\circ}$ and for each $\phi$ the incidence angle $(\theta)$ is varied from $0^{\circ}$ to $60^{\circ}$ in steps of $15^{\circ}$ (see Fig. 1(c)). Most authors studying the angular stability of symmetric unit-cells only consider the case of $\phi=0$ and sweep $\theta$. However, it is important to point out that although the structure behaves identically for any polarization angle under normal incidence, it could be not the case when oblique incidence is tackled. Moreover, in order to analyze the angular stability two parameters are considered: stable bandwidth $(B s)$ (minimum bandwidth under which the structure behaves as an AMC regardless the polarization and the incidence angle of the incident plane wave) and resonance frequency deviation (Af) (maximum deviation of the resonance frequency) [13]. The narrowest stable bandwidth (711 $\mathrm{MHz}$ ) occurs for TE-polarization with $\phi=75^{\circ}$ (Fig. 2a). In Fig. 2b, the TM-polarization with $\phi=0^{\circ}$ is shown; this is the case in which the maximum frequency deviation $(529 \mathrm{MHz})$ takes place.
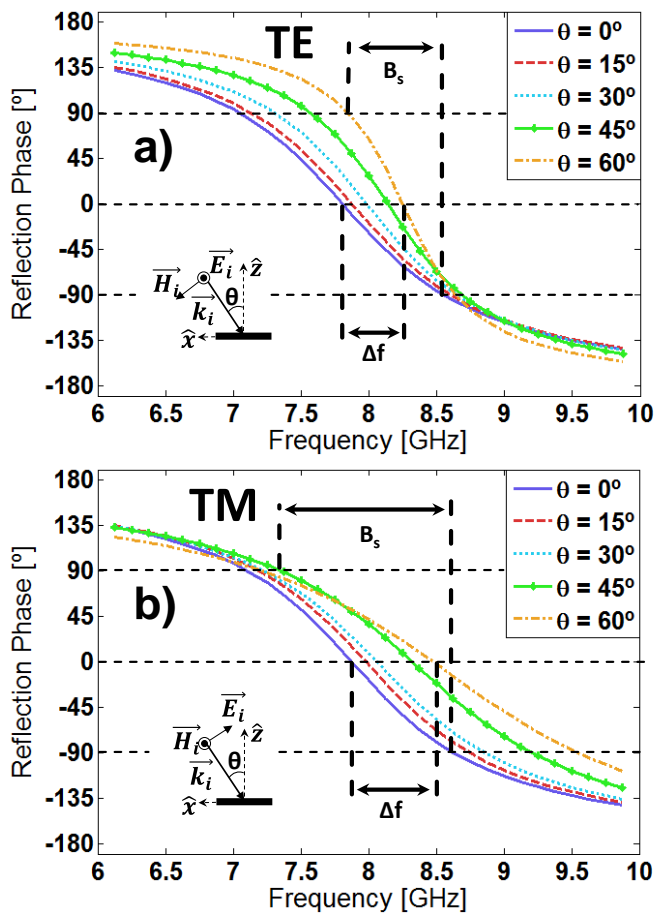

Fig. 2. Reflection coefficient phase for (a) TE-polarization $\left(\phi=75^{\circ}\right)$ and (b) TM-polarization $\left(\phi=0^{\circ}\right)$.

The LUC based AMC resonates at $7.79 \mathrm{GHz}$ with a bandwidth of $1508 \mathrm{MHz}(19.37 \%)$ at normal incidence. The results of the complete angular stability analysis are gathered numerically in TABLE I. where the aforementioned worst cases for stable bandwidth and frequency deviation are highlighted in bold orange. These values obviously coincide with the ones depicted in Fig. 2.

TABLE I. ANGULAR STABILITY RESULTS FOR LUC BASED AMC

\begin{tabular}{|c|c|c|c|c|c|c|c|c|}
\hline & \multicolumn{4}{|c|}{ TE } & \multicolumn{4}{|c|}{ TM } \\
\hline$\Phi=0$ & 425 & 5.31 & 728 & 8.91 & 529 & 6.57 & 1342 & 17.07 \\
\hline$\Phi=30$ & 428 & 5.34 & 728 & 8.90 & 525 & 6.53 & 1353 & 17.25 \\
\hline$\Phi=45$ & 439 & 5.47 & 720 & 8.78 & 461 & 5.73 & 1314 & 16.60 \\
\hline$\Phi=60$ & 383 & 4.77 & 774 & 9.45 & 436 & 5.41 & 1397 & 17.71 \\
\hline
\end{tabular}

Nowadays, designers have to face some constraints difficult to meet. In actual applications there is a limited space for the device arrangement (in this case the AMC), so the miniaturization (introducing as many unit-cells as possible in the allocated space and using a reduced dielectric thickness) plays a fundamental role in the design. Moreover, this must be achieved while preserving the bandwidth and the resonance frequency specifications. As one can imagine this is not an easy task at all.

The aim of this work is to design an AMC based on the previously described LUC, with a $5.8 \mathrm{GHz}$ resonance frequency and a proper bandwidth (wide enough for the common intended applications), preserving the dielectric substrate characteristics (RO4003C and thickness $1.524 \mathrm{~mm}$ ) and without 
increasing the unit-cell dimensions $(p=7.3 \mathrm{~mm})$. Moreover, due to the fact that this AMC is not angularly stable, an improvement on its angular stability will be sought. Analyzing the expression of the AMC's resonance frequency (4), the only way to reduce its value, without altering the periodicity and/or the dielectric characteristics, is by increasing the grid inductance. However, this is not new. What is really new is the study focused on the influence of introducing lumped inductors on the AMCs' angular stability.

\section{A. Further explanation of the loop-based AMC behavior and some guidelines regarding the lumped inductors choice.}

Analyzing the loop-based AMC's equivalent circuit and its resonance frequencies given by (3) and (4) several remarks can be done. The behavior of the structure mainly depends on the ratio of the grid and the dielectric inductances' values ( $L_{g}$ and $L_{d}$, respectively). The latter is directly proportional to the thickness of the dielectric provided an electrically thin dielectric is considered [13]. These values $\left(L_{g}\right.$ and $\left.L_{d}\right)$ determine the frequency location of the zero and the pole of the AMC's equivalent (surface) impedance $\left(Z_{s}\right)$ (see expressions (3) and (4)). Therefore, the AMC behaves differently in terms of resonance frequency, angular stability and bandwidth for different values of $L_{g}$.

On the one hand, if $L_{d}>L_{g}$, the frequency location of the zero and the pole of $Z_{s}$ are far away enough. Thus, the zero does not perturb the behavior of the structure and the AMC's resonance frequency is properly described by (4). Consequently, when the dielectric thickness increases $\left(\uparrow L_{d}\right)$ the grid inductance must decrease $\left(\downarrow L_{g}\right)$ to keep the resonance frequency.

On the other hand, if $L_{d} \ll L_{g}$, the pole and the zero frequencies are very close to each other. Therefore, an overlap of both resonant effects may occur in the structure and hence a peculiar behavior is observed. In this case, decreasing the dielectric thickness $\left(\downarrow L_{d}\right)$ also implies the reduction of the grid inductance $\left(\downarrow L_{g}\right)$ in order to maintain the resonance frequency.

Since both cases entail decreasing the grid inductance $\left(L_{g}\right)$ in order to preserve the AMC's resonance frequency, an upper limit for $L_{g}$ value can be defined coinciding with a similar value for both inductances $\left(L_{d} \sim L_{g}\right)$.

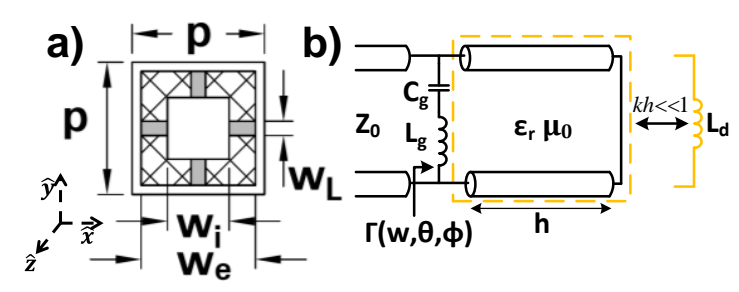

Fig. 3. (a) Geometry of LIUC (top view). (b) Equivalent circuit model of LIUC based AMC.

In this work the grid inductance $\left(L_{g}\right)$ is tailored introducing lumped inductors. Thus, a new square loop-based unit-cell (LIUC) including lumped inductors (of L value) soldered on its unit-cell's metallization geometry, is proposed (Fig. 3a). In order to analyze only the effect of the lumped inductors, the LIUC is identical to the LUC (Fig. 1a) except for the introduction of four lumped inductors. Both LIUC and LUC based AMCs can be modeled using the same equivalent circuit components (Fig. $3 \mathrm{~b}$ and Fig. 1b), provided their values are considered properly in each case. The width of the loop metallization strips was wisely designated in the LUC based AMC (width $\left.=\left(\left(w_{e}-w_{i}\right) / 2\right)=1.45 \mathrm{~mm}\right)$ so as to accurately arrange the inductors of 0805 size [27] $\left(w_{L}=0.8 \mathrm{~mm}\right)$. The lumped inductor values are appropriately chosen to get an AMC's resonance frequency at $5.8 \mathrm{GHz}$. Considering a dielectric thickness of $1.524 \mathrm{~mm}$ and supposing normal incidence and an electric field along $\mathrm{x}$-axis, the grid capacitance $\left(C_{g}\right)$ can be estimated using the formulation proposed in [28] for a grid of conductive strips which gives a $0.203 \mathrm{pF}$ grid capacitance value. The grid inductance $\left(L_{g}\right)$ can be modeled by a parallel connection of the inductances yielded by the top and bottom parts of the unit-cell (the metallic strip inductance in series with the lumped inductor, $\left(L_{1}\right)$ ). Using the formulation proposed in [29], concerning the inductance calculation for metallic strips on grounded dielectrics, the inductance given by the considered metallic strips $\left(L_{m}\right)$ which remains after the introduction of the lumped inductors can be estimated, given a value of $L_{m}=1.61 \mathrm{nH}$ for the dielectric thickness under consideration $(1.524 \mathrm{~mm})$. The inductances of the top and bottom parts of the unit-cell are equal (so $L_{g}=L_{1} / 2$ ) and its value is $L_{1}=L_{m}+L$. On the other hand, the value of $L_{d}$ for electrically thin dielectrics can be approximated by $L_{d} \sim \mu h$, where $\mu$ is the dielectric permeability and $h$ its thickness [13]. For the dielectric thickness considered here $(1.524 \mathrm{~mm})$, the dielectric inductance is $L_{d}=1.92 \mathrm{nH}$. Therefore taken into consideration that the resonance frequency (4) must be $5.8 \mathrm{GHz}$ the value of $\mathrm{L}$ can be solved and it should be $\mathrm{L}=1.99 \mathrm{nH}$. This value is very close to 
the one needed in electromagnetic simulation $(1.8 \mathrm{nH})$ to get the desired resonance frequency. Although more complex models, which fit more accurately the AMC's behavior, can be used, the circuit model intuitive comprehension is lost and a full-wave electromagnetic analysis is usually preferable. Thereupon, even though highly order modes are sometimes considered in the equivalent circuit model when the dielectric thickness is much smaller than the unit-cell periodicity [30], the model proposed here is insightful for the analysis carried out in this article.

TABLE II. AMC BEHAVIOR WITHOUT AND WITH LUMPED INDUCTANCES FOR DIFFERENT DIELECTRIC THICKNESSES

\begin{tabular}{cccc}
\hline $\begin{array}{c}\text { Thickness } \\
(\mathbf{m m})\end{array}$ & $\begin{array}{c}\boldsymbol{f}_{\boldsymbol{L U C}} \\
(\boldsymbol{G H z})\end{array}$ & $\begin{array}{c}\boldsymbol{f}_{\boldsymbol{L I U C}} \\
(\boldsymbol{G H z})\end{array}$ & $\begin{array}{c}\boldsymbol{L} @ \mathbf{5 . 8 G H z} \\
(\boldsymbol{n H})\end{array}$ \\
\hline 2.2 & 6.74 & 5.44 & 1.15 \\
1.8 & 7.34 & 5.63 & 1.62 \\
1.524 & 7.79 & 5.78 & 1.8 \\
1.3 & 8.20 & 5.84 & $\mathbf{1 . 8 6}$ \\
1.2 & 8.43 & 5.83 & $\mathbf{1 . 8 6}$ \\
1 & 8.78 & $\mathbf{5 . 8 4}$ & $\mathbf{1 . 8 6}$ \\
0.9 & 8.94 & 5.81 & $\mathbf{1 . 8 6}$ \\
0.8 & 9.06 & 5.77 & 1.78 \\
0.7 & 9.17 & 5.67 & 1.70 \\
0.6 & 9.28 & 5.46 & 1.55 \\
0.5 & 9.41 & 5.30 & 1.38 \\
0.4 & 9.45 & 5.05 & 1.19 \\
0.3 & 9.51 & 4.68 & 0.96 \\
0.2 & 9.46 & 4.06 & 0.65 \\
0.1 & 9.31 & 3.06 & 0.35 \\
\hline
\end{tabular}

With the aim of checking the previous remarks about the AMC's behavior for different ratios of $L_{d}$ and $L_{g}$, the dielectric thickness is varied (from $2.2 \mathrm{~mm}$ to $0.1 \mathrm{~mm}$ ). Thence, dielectric inductance's values greater and smaller than the grid inductance's one are obtained. For each dielectric thickness, electromagnetic simulations were carried out for both LUC and LIUC based AMCs. TABLE II. gathers the following results: the resonance frequency of the LUC based $\operatorname{AMC}\left(f_{L U C}\right)$, the resonance frequency of the LIUC based AMC with inductors of value $1.8 \mathrm{nH}\left(f_{\text {LIUC }}\right)$ and the value of the inductors needed to reach a $5.8 \mathrm{GHz}$ resonance frequency in the LIUC based AMC $(L @ 5.8 \mathrm{GHz})$.

For the LUC based AMC (second column of the table) it is clear that when the dielectric thickness decreases $\left(\downarrow L_{d}\right)$ the resonance frequency increases. This behavior is due to the fact that the AMC's resonance frequency is well ruled by (4), since $L_{d}>L_{g}$. However, for very thin dielectric slabs (bold blue values), the value of $L_{d}$ is much smaller than the one of $L_{g}$ (the zero and the pole of the equivalent (surface) impedance are close to each other), bringing about changes in the behavior of the resonance frequency.

For LIUC based AMC with lumped inductors of $1.8 \mathrm{nH}$ (third column of the table) it is observed that as the dielectric thickness decreases the resonance frequency increases (AMC resonance behavior ruled by (4) since $L_{d}>L_{g}$ ). Following this tendency up to a range of dielectric thickness values where the resonance frequency keeps nearly constant (bold green values $\left(L_{d} \sim L_{g}\right)$ ). Further decreasing the dielectric thickness entails a continuous shift in the AMC's resonance frequency to lower frequencies. This phenomenon is attributed to the zero and the pole frequencies overlap in the AMC's equivalent (surface) impedance $\left(L_{d} \ll L_{g}\right)$.

According to the previous statements the required lumped inductor values to preserve the resonance frequency at $5.8 \mathrm{GHz}$ as the dielectric thickness decreases are presented in the fourth column of the table. For thicker dielectric slabs $\left(L_{d}>L_{g}\right)$, the expression (4) describes the AMC's resonance frequency behavior, so the lumped inductor value should be increased as the dielectric thickness decreases, up to a range of dielectric thicknesses where the dielectric and grid inductances are similar $\left(L_{d} \sim L_{g}\right.$ (bold green values)). In this latter region, the inductor values should be kept constant to preserve the resonance frequency. Further reducing the dielectric thickness $\left(L_{d} \ll L_{g}\right)$ causes the zero and the pole of the AMC's equivalent (surface) impedance overlap and the lumped inductor values should be decreased to preserve the resonance frequency.

\section{B. Comparison between LUC and LIUC based AMCs for different commercial dielectric thicknesses regarding angular stability.}

Now with the previous remarks in mind, the effect of introducing inductors in the unit-cell's metallization geometry will be analyzed for different dielectric thicknesses (TABLE III. ). Firstly, it must 
be stated again that for each thickness the LUC and LIUC based AMCs are identical except for the introduction of lumped inductors in the latter one. In all the cases tackled here, the resonance frequency of the LUC based AMC will be above $5.8 \mathrm{GHz}$, due to the considered dielectric and the periodicity preservation. The inductor values in the LIUC based AMC will be chosen to shift the resonance frequency up to $5.8 \mathrm{GHz}$ to meet the aforementioned requirements.

From now on, only the worst cases of polarization $(\phi)$ will be presented. Indeed, these cases can be considered to be the AMC's operating specifications (narrowest stable bandwidth and maximum resonance frequency deviation). For each dielectric thickness the same simulation procedure as the one used for the LUC based AMC is followed.

TABLE III. ANGULAR STABILITY RESULTS FOR LUC AND LIUC BASED AMC WITH DIFFERENT DIELECTRIC THICKNESSES

\begin{tabular}{ccccc}
\hline & $\begin{array}{c}\boldsymbol{A f} \\
(\boldsymbol{M H z})\end{array}$ & $\begin{array}{c}\boldsymbol{A f} \\
(\boldsymbol{\%})\end{array}$ & $\begin{array}{c}\boldsymbol{B s} \\
(\boldsymbol{M H z})\end{array}$ & $\begin{array}{c}\boldsymbol{B} \boldsymbol{( \boldsymbol { \% } )} \\
\left(\begin{array}{c}\boldsymbol{\%} \\
\end{array}\right.\end{array}$ \\
\hline LUC (1.524 mm) & 529 & 6.57 & 711 & 8.67 \\
LIUC (1.524 mm) & 186 & 3.17 & 220 & 3.82 \\
Difference (1.524 mm) & -343 & -51.75 & -491 & -55.94 \\
LUC (0.762 mm) & 345 & 3.74 & 255 & 2.73 \\
LIUC (0.762 mm) & 71 & 1.21 & 79 & 1.36 \\
Difference (0.762 mm) & -274 & -67.65 & -176 & -50.20 \\
\hline
\end{tabular}

As it was previously stated, for dielectric thickness of $1.524 \mathrm{~mm}$, lumped inductors of $1.8 \mathrm{nH}$ are introduced in the LIUC based AMC so that it resonates at $5.8 \mathrm{GHz}$. A bandwidth of $450 \mathrm{MHz}(7.8 \%)$ at normal incidence is obtained in this LIUC based AMC. The narrowest stable bandwidth occurs for TEpolarization $(220 \mathrm{MHz})$ and the higher frequency deviation arise for TM-polarization $(186 \mathrm{MHz})$, in both cases the polarization angle coincides $\left(\phi=0^{\circ}\right)$. Comparing the results of the LUC and LIUC based AMCs, several remarks can be highlighted. First of all, the LIUC based AMC's resonance frequency is $25.55 \%$ lower than the LUC based AMC's one, preserving the unit-cell periodicity and the dielectric characteristics. Although the stable bandwidth is narrowed, the frequency deviation is reduced by $51.75 \%$ from the one in the LUC based AMC.

Considering a dielectric thickness of $0.762 \mathrm{~mm}$ the corresponding resonance frequencies are $9.09 \mathrm{GHz}$ and $5.8 \mathrm{GHz}$ and the bandwidth are $815 \mathrm{MHz}$ and $166 \mathrm{MHz}$ at normal incidence for the LUC and LIUC based AMCs, respectively. Inductances of value $1.7 \mathrm{nH}$ are needed in the LIUC based AMC to shift down the resonance frequency up to $5.8 \mathrm{GHz}$. Thus, a decrease in the resonance frequency of $36.2 \%$ is achieved. Concerning the AMC's angular stability, the frequency deviation is further reduced $(67.65 \%)$ if it is compared with the previous case (thicker dielectric AMC (1.524mm)). Moreover, the stable bandwidth is also narrowed but to a lesser extent (50.2\%) compared to the previous analyzed dielectric thickness.

Therefore, as one can see, the achieved improvements in the angular stability and miniaturization are noticeable at the expense of a narrowing in the stable bandwidth. However, the latter is not a problem provided the application's bandwidth requirements are satisfied. Moreover, the frequency deviation reduction is essential in applications requiring narrow bandwidth to maintain the AMC's operation band.

On the other hand, if the LUC based AMC is designed to resonate at $5.8 \mathrm{GHz}$ the unit-cell periodicity has to be increased. In this case not only the frequency deviation is increased but also the stable bandwidth is narrowed if compared to the LIUC based AMC for each of the analyzed dielectric thicknesses.

\section{Range of dielectric thicknesses where this method can be applied preserving the resonance frequency}

In this section, the range of dielectric thicknesses for which introducing lumped inductors improves the angular stability of square loop-based AMCs, keeping the resonance frequency, will be discussed. Thus, an upper and a lower dielectric thickness values should be defined.

On the one hand, concerning the upper dielectric thickness and considering $L_{d}>L_{g}$, the higher frequency deviation will appear under TM-polarized incident wave. Indeed, the thicker the dielectric is, the higher the frequency deviation and hence, a greater value of grid inductance is necessary to reduce this frequency shift. However, as it was shown in Section II when $L_{d}>L_{g}$, smaller values of lumped inductors are necessary to preserve the resonance frequency as the dielectric thickness increases. Therefore, considering that the resonance frequency must be preserved, the value of $L_{g}$ (and hence the one of the lumped inductors) must decrease as the dielectric thickness increases. Then, the improvements in the AMCs' angular stability are negligible since the value of these lumped inductors should be small to preserve the resonance frequency. Consequently, the upper dielectric thickness limit is reached once the introduction of lumped inductors no longer improves the AMC's angular stability. However, thick 
dielectrics are not of interest in actual applications due to general profile constrains. Thus, in order to build up a low profile AMC, its thickness is commonly considered to be less than $\lambda / 10$. Moreover, grounded dielectric slabs with thickness greater than $\lambda / 4$ can no longer be modeled as inductances.

On the other hand, there is no theoretical lower limit on the dielectric thickness, since for $L_{g} \gg L_{d}$ introducing lumped inductors always reduce the frequency deviation as it will be shown later from simulation results. Moreover, there is a dielectric thickness from which the stable bandwidth in the LIUC based AMC is wider than the one of the LUC based AMC. This is possible due to the fact that the reduction of the frequency deviation thanks to the lumped inductor introduction compensates the narrowing in bandwidth originated by the shifting down of the resonance frequency. Therefore, the lower limit in the dielectric thickness will be given by the desired operation bandwidth for the intended application provided that a realizable AMC can be obtained.

TABLE IV. is presented with the aim of clarify the previous statements. Two zones are clearly visible. In the first one (thickness from 1.8 to $2.1 \mathrm{~mm}$ ), as the thickness increases the improvement in the frequency deviation is smaller, since small lumped inductors are introduced to keep the resonance frequency at $5.8 \mathrm{GHz}$. On the other hand, in the second zone (from 0.508 to $0.305 \mathrm{~mm}$ ) the reduction on the dielectric thickness improves relatively both the frequency deviation and the stable bandwidth. The frequency deviation is reduced as far as $74.67 \%$ and the stable bandwidth is relatively widened by $56.16 \%$ for dielectric thickness of $0.305 \mathrm{~mm}$.

TABLE IV. ANGULAR STABILITY RESULTS FOR LUC AND LIUC BASED AMCS WITH OTHER DIELECTRIC THICKNESSES

\begin{tabular}{ccccc}
\hline & $\begin{array}{c}\boldsymbol{A f} \\
(\boldsymbol{M H} \boldsymbol{z})\end{array}$ & $\begin{array}{c}\boldsymbol{A f} \\
(\boldsymbol{\%})\end{array}$ & $\begin{array}{c}\boldsymbol{B s} \\
(\boldsymbol{M H} \boldsymbol{z})\end{array}$ & $\begin{array}{c}\boldsymbol{B s} \\
(\boldsymbol{\%})\end{array}$ \\
\hline LUC $(2.1 \mathrm{~mm})$ & 613 & 8.48 & 880 & 11.92 \\
LIUC $(2.1 \mathrm{~mm}, 1.3 \mathrm{nH})$ & 445 & 7.40 & 434 & 7.24 \\
Difference $(2.1 \mathrm{~mm})$ & -168 & -12.74 & -446 & -39.26 \\
LUC $(1.9 \mathrm{~mm})$ & 598 & 7.92 & 849 & 11.06 \\
LIUC $(1.9 \mathrm{~mm}, 1.4 \mathrm{nH})$ & 362 & 6.04 & 357 & 6.10 \\
Difference $(1.9 \mathrm{~mm})$ & -0.236 & -23.74 & -492 & -44.85 \\
LUC $(1.8 \mathrm{~mm})$ & 607 & 7.95 & 799 & 10.31 \\
LIUC $(1.8 \mathrm{~mm}, 1.5 \mathrm{nH})$ & 312 & 5.24 & 319 & 5.46 \\
Difference $(1.8 \mathrm{~mm})$ & -295 & -34.10 & -480 & -47.04 \\
\hline & & & & \\
\hline LUC $(0.508 \mathrm{~mm})$ & 194 & 2.05 & 177 & 1.86 \\
LIUC $(0.508 \mathrm{~mm}, 1.4 \mathrm{nH})$ & 40 & 0.69 & 59 & 1.02 \\
Difference $(0.508 \mathrm{~mm})$ & -154 & -66.34 & -118 & -44.62 \\
LUC $(0.406 \mathrm{~mm})$ & 190 & 2 & 105 & 1.08 \\
LIUC $(0.406 \mathrm{~mm}, 1.2 \mathrm{nH})$ & 32 & 0.55 & 60 & 1.03 \\
Difference $(0.406 \mathrm{~mm})$ & -158 & -72.5 & -0.045 & -4.63 \\
LUC $(0.305 \mathrm{~mm})$ & 144 & 1.50 & 70 & 0.73 \\
LIUC $(0.305 \mathrm{~mm}, 1 \mathrm{nH})$ & 22 & 0.38 & 66 & 1.14 \\
Difference $(0.305 \mathrm{~mm})$ & -122 & -74.67 & -4 & +56.16 \\
\hline & & & &
\end{tabular}

\section{Comparison with other literature contributions}

This section will be focused on comparing the results of this article with previous literature contributions. Although there are no many articles in which the AMC's angular stability is analyzed and even less where the analysis is carried out under different polarization angles $(\phi)$, some comparisons can be made.

TABLE V. shows the AMC characteristics presented in several literature contributions. In this table the following data are gathered: the dielectric slab properties (relative dielectric permittivity and thickness), the absolute and relative bandwidth at normal incidence, the resonance frequency and the worst cases for the absolute and relative values of the frequency deviation and the stable bandwidth of the AMCs reported in the different contributions. The striped table cells refer to articles in which only polarization angles corresponding to $\phi=0^{\circ}$ are analyzed. In order to consider a prototype with similar characteristics to the ones found in the literature contributions, the LIUC based AMC with a dielectric thickness of $1.524 \mathrm{~mm}$ is used to carry out the comparisons.

TABLE V. COMPARISON WITH OTHER ARTICLES

\begin{tabular}{|c|c|c|c|c|c|c|c|c|c|}
\hline & $\begin{array}{c}\text { Material } \\
\left(\varepsilon_{r}\right)\end{array}$ & $\begin{array}{c}\text { Thickness } \\
(\mathrm{mm})\end{array}$ & $\begin{array}{c}B W \\
(M H z)\end{array}$ & $\begin{array}{l}B W \\
(\%) \\
\end{array}$ & $\begin{array}{c}f_{r} \\
(G H z)\end{array}$ & $\begin{array}{c}A f \\
(\boldsymbol{M H z})\end{array}$ & $\begin{array}{l}A f \\
(\%)\end{array}$ & $\begin{array}{c}\text { Bs } \\
(\mathbf{M H z})\end{array}$ & $\begin{array}{c}B s \\
(\%)\end{array}$ \\
\hline $\begin{array}{c}{[21]} \\
\text { Mushroom }\end{array}$ & 2.2 & 6.5 & - & - & 5 & 1000 & 20 & - & - \\
\hline $\begin{array}{c}{[21]} \\
\text { SS }\end{array}$ & 2.2 & 6.3 & - & - & 8.75 & 700 & 8 & - & -2 \\
\hline
\end{tabular}




\begin{tabular}{|c|c|c|c|c|c|c|c|c|c|}
\hline & $\begin{array}{c}\text { Material } \\
\left(\varepsilon_{r}\right)\end{array}$ & $\begin{array}{c}\text { Thickness } \\
(\mathrm{mm})\end{array}$ & $\begin{array}{c}B W \\
(M H z)\end{array}$ & $\begin{array}{l}B W \\
(\%)\end{array}$ & $\begin{array}{c}f_{r} \\
(\boldsymbol{G H z})\end{array}$ & $\begin{array}{c}A f \\
(M H z)\end{array}$ & $\begin{array}{l}A f \\
(\%)\end{array}$ & $\begin{array}{c}B s \\
(M H z)\end{array}$ & $\begin{array}{c}B s \\
(\%)\end{array}$ \\
\hline $\begin{array}{l}\text { [21] } \\
\text { SSL }\end{array}$ & 2.2 & 4.7 & - & - & 6.25 & 500 & 8 & - & - \\
\hline $\begin{array}{c}{[4]} \\
\text { SRG-DiNV }\end{array}$ & 2.7-j0.01 & 6 & $869 \div$ & 22 & 3.95 & 170 & 4.3 & - & - \\
\hline $\begin{array}{c}\text { [4] } \\
\text { SRG-DiV }\end{array}$ & 2.7-j0.01 & 6 & - & - & 3.95 & 474 & 12 & - & - \\
\hline $\begin{array}{c}{[4]} \\
\text { JC-DiV }\end{array}$ & $2.7-\mathrm{j} 0.01$ & 6 & - & - & 3.95 & 750 & $19-$ & - & - \\
\hline $\begin{array}{c}{[4]} \\
\text { JC-DiNV }\end{array}$ & $\begin{array}{l}\text { Alumina } \\
(\mathbf{9} . \mathbf{3}-11.5)\end{array}$ & 0.653 & -1 & - & 15 & 1050 & 7 & - & - \\
\hline $\begin{array}{c}{[22]} \\
\text { Design I }\end{array}$ & 6.15 & 2.54 & 4560 & 45.41 & 10.04 & 630 & 6.27 & - & - \\
\hline $\begin{array}{c}{[22]} \\
\text { Design II }\end{array}$ & 6.15 & 2.54 & 4430 & $\mathbf{4 5 . 4 8}$ & 9.74 & 629 & 6.46 & - & -1 \\
\hline $\begin{array}{c}{[24]} \\
\text { Hexagon }\end{array}$ & 3.38 & 1.524 & 3020 & 23.5 & 12.85 & 850 & 6.61 & - & - \\
\hline $\begin{array}{c}{[24]} \\
\text { Square }\end{array}$ & 3.38 & 1.524 & 1700 & 13.3 & 12.85 & 760 & 5.91 & - & - \\
\hline $\begin{array}{c}{[13]} \\
\text { HI_0.6 }\end{array}$ & 3.38 & 1.524 & 690 & 12 & 5.78 & 382 & 6.4 & 182 & 3 \\
\hline $\begin{array}{c}{[13]} \\
\text { SI_0.6 }\end{array}$ & 3.38 & 1.524 & 578 & 10.13 & 5.7 & 362 & 6.1 & 69 & 1.2 \\
\hline Proposed AMC & 3.38 & 1.524 & 450 & 7.8 & 5.8 & 186 & 3.17 & 220 & 3.82 \\
\hline
\end{tabular}

* The bandwidth is measured for $\pm 45^{\circ}$

As one can see from the table, the proposed AMC is the one with the smallest relative frequency deviation. However, in order to carry out a fair comparison the AMCs should be in the same frequency band and identical dielectric characteristics must be used. Therefore, the AMC proposed in [13] with a similar gap $(1.2 \mathrm{~mm})$ between unit-cells to the one proposed in this paper $(1 \mathrm{~mm})$ is a good candidate. It is observed that the proposed AMC does not only reduce the frequency deviation by a half, but also increases the stable bandwidth from both square and hexagonal loop-based AMCs (Sl_0.6 and Hl_0.6) presented in [13]. In addition, although it is not the aim of the proposed AMC, its unit-cell area $\left(0.53 \mathrm{~cm}^{2}\right)$ is much smaller than the unit-cell area of the AMCs proposed in [13] $\left(0.82 \mathrm{~cm}^{2}\right.$ and $0.88 \mathrm{~cm}^{2}$ for S1_0.6 and Hl_0.6, respectively).

\section{Conclusion}

The introduction of lumped inductors to improve the angular stability of AMCs has been explained and justified by both an equivalent circuit model and electromagnetic simulations. A non-angular stable AMC is selected in order to study the improvements introduced by the lumped inductors. Therefore, a square loop-based AMC is chosen. First of all, the behavior of this AMC, using an equivalent circuit model, is analyzed and explained in order to give some guidelines in the choice of the lumped inductors value. An upper grid inductance limit is provided from which the resonance frequency could not be preserved. Then, the AMC's angular stability without and with lumped inductors is studied for several dielectric slab thicknesses with the aim of analyzing the improvements introduced by the latter. A range of validity for the dielectric thickness where the proposed technique can be applied, show that the limitations are more in the application specifications than in the introduction of the lumped inductors. Finally, comparison of the proposed AMC and the ones found in literature contributions are carried out. These comparisons confirm that the technique introduced here to improve the AMCs' angular stability outperforms the ones presented in the literature.

Consequently, it has been shown that the proposed AMC with lumped inductors can not only miniaturize the structure but also reduce the resonance frequency deviation and widen the stable bandwidth for whichever angle of incidence and polarization.

The technique introduced here is not only valid for AMCs comprising square loop-based unit-cells but also to no matter which series resonant grid based AMC, preserving the aforementioned advantages (fitting to the design constraints, miniaturization of the unit-cell and angular stability improvements).

Moreover, other studies have been conducted by these authors introducing lumped capacitors on patch-based unit-cells concluding also in angular stability improvement. However, patch-based unit-cells are not as stable as the loop-based ones. 


\section{Acknowledgment}

This work was supported by the Gobierno del Principado de Asturias/FEDER under Project GRUPIN14-114 and by the Ministerio de Economía y Competitividad under Project TEC2014-54005-P (MIRIIEM).

\section{References}

[1] M. Hosseini, A. Pirhadi, and M. Hakkak, "A novel AMC with little sensitivity to the angle of incidence using 2-layer Jerusalem cross FSS," Progress In Electromagnetics Research, vol. 64, pp. 43-51, 2006. http://dx.doi.org/10.2528/PIER06061301.

[2] M. Hosseini, A. Pirhadi, and M. Hakkak, "Compact angularly stable AMCs utilizing skewed cross-shaped FSSS," Microwave and Optical Technology Letters, vol. 49, no. 4, pp. 781-786, 2007. http://dx.doi.org/10.1002/mop.22280.

[3] M. Hosseini, A. Pirhadi, and M. Hakkak, "Design of a non-uniform high Impedance surface for a low profile antenna," Journal of Electromagnetic Waves and Applications, vol. 20, no. 11, pp. 1455-1464, Jan. 2006. http://dx.doi.org/10.1163/156939306779274291.

[4] C. R. Simovski, P. de Maagt, and I. V. Melchakova, "High-impedance surfaces having stable resonance with respect to polarization and incidence angle," IEEE Transactions on Antennas and Propagation, vol. 53, no. 3, pp. 908-914, Mar. 2005. http://dx.doi.org/10.1109/TAP.2004.842598.

[5] D. Sievenpiper, L. Zhang, R. F. J. Broas, N. G. Alexopolous, and E. Yablonovitch, "High-impedance electromagnetic surfaces with a forbidden frequency band," IEEE Transactions on Microwave Theory and Techniques, vol. 47, no. 11, pp. 2059-2074, 1999. http://dx.doi.org/10.1109/22.798001.

[6] A. P. Feresidis, G. Goussetis, S. Wang, and J. C. Vardaxoglou, "Artificial magnetic conductor surfaces and their application to low-profile high-gain planar antennas," IEEE Transactions on Antennas and Propagation, vol. 53, no. 1, pp. 209-215, Jan. 2005. http://dx.doi.org/10.1109/TAP.2004.840528.

[7] M. E. de Cos, Y. Alvarez Lopez, and F. Las-Heras, "A novel approach for RCS reduction using a combination of artificial magnetic conductors," Progress In Electromagnetics Research, vol. 107, pp. 147-159, 2010. http://dx.doi.org/10.2528/PIER10060402.

[8] S. Lee, N. Kim and S-Y. Rhee, "Design of novel artificial magnetic conductor as reflector and its SAR analysis". In Progress In Electromagnetics Research Symposium Proceedings, Moscow, Russia, 2012, pp. 329 - 333.

[9] V. G. M. Annamdas and C. K. Soh, "Contactless load monitoring in near-field with surface localized spoof plasmons-A new breed of metamaterials for health of engineering structures," Sensors and Actuators A: Physical, vol. 244, pp. 156-165, 2016. http://dx.doi.org/10.1016/j.sna.2016.04.037.

[10] S. Papantonis, N. M. Ridler, and S. Lucyszyn, "Rectangular waveguide enabling technology using holey surfaces and wire media metamaterials," Sensors and Actuators A: Physical, vol. 209, pp. 1-8, 2014. http://dx.doi.org/10.1016/j.sna.2014.01.005.

[11] F. Capolino, Theory and phenomena of metamaterials, 1st ed. CRC press, 2009.

[12] D.M. Pozar, Microwave engineering, 4rd ed. John Wiley \& Sons, 2009.

[13] M. E. de Cos and F. Las-Heras, "On the advantages of loop-based unit-cell's metallization regarding the angular stability of artificial magnetic conductors," Applied Physics A, vol. 118, no. 2, pp. 699-708, 2014. http://dx.doi.org/10.1007/s00339-0148782-8.

[14] A. Feresidis, G. Goussetis, and J. Vardaxoglou, "Metallodielectric arrays without vias as artificial magnetic conductors and electromagnetic band gap surfaces, " IEEE Antennas and Propagation Society Symposium, 2004. http://dx.doi.org/10.1109/APS.2004.1330388.

[15] W. A. Goddard, D. W. Brenner, S. E. Lyshevski, and G. J. Lafrate, Handbook of nanoscience, engineering, and technology, 3rd ed. CRC Press, 2012.

[16] M. Hosseini and M. Hakkak, "Characteristics estimation for Jerusalem cross-based artificial magnetic conductors," IEEE Antennas and Wireless Propagation Letters, vol. 7, pp. 58-61, 2008. http://dx.doi.org/10.1109/LAWP.2008.917605.

[17] F. Yang and Y. Rahmat-Samii, "Reflection phase characterizations of the EBG ground plane for low profile wire antenna applications," IEEE Transactions on Antennas and Propagation, vol. 51, no. 10, pp. 2691-2703, 2003. http://dx.doi.org/10.1109/TAP.2003.817559.

[18] M. Abu, E. E. Hussin, A. R. Othman, N. M. Yatim, F. M. Johar, and R. F. Munawar, "Design of stacked wafers AMC at 920 MHz for metallic object detection in RFID application," 2013 IEEE Symposium on Wireless Technology \& Applications (ISWTA), 2013. http://dx.doi.org/10.1109/ISWTA.2013.6688778.

[19] J. Mcvay, A. Hoorfar, and N. Engheta, "Bandwidth enhancement and polarization dependence elimination of space-filling curve artificial magnetic conductors," 2007 Asia-Pacific Microwave Conference, 2007. http://dx.doi.org/10.1109/APMC.2007.4555127.

[20] O. Luukkonen, C. R. Simovski, and S. A. Tretyakov, "Grounded uniaxial material slabs as magnetic conductors," Progress In Electromagnetics Research B, vol. 15, pp. 267-283, 2009. http://dx.doi.org/10.2528/PIERB0905070.

[21] C. Simovski, P. D. Maagt, S. Tretyakov, M. Paquay, and A. Sochava, "Angular stabilisation of resonant frequency of artificial magnetic conductors for TE-incidence," Electronics Letters, vol. 40, no. 2, p. 92-93, 2004. http://dx.doi.org/10.1049/el:20040064.

[22] G. Gampala and A.B. Yakovlev, "Artificial magnetic conductors for wideband antenna applications," in 2007 Proceedings of ACES Conference, Verona, 2007, pp. 242-247.

[23] N. Christopoulos, G. Goussetis, A. P. Feresidis and J. C. Vardaxoglou, "Metamaterials with multiband AMC and EBG properties," 2005 European Microwave Conference, 2005. http://dx.doi.org/10.1109/EUMC.2005.1608883.

[24] M. E. de Cos and F. Las-Heras, "Multi-band artificial magnetic conductors with high angular stability," 2013 7th European Conference on Antennas and Propagation (EuCAP), Gothenburg, 2013, pp. 2510-2512. 
[25] H. Liu, K. L. Ford and R. J. Langley, "Miniaturised artificial magnetic conductor design using lumped reactive components," Electronics Letters, vol. 45, no. 6, pp. 294-295, March 12 2009. http://dx.doi.org/10.1049/el.2009.3369.

[26] M. A. Aziz, M. M. Shukor, M. Suaidi, B. H. Ahmad, M. F. Johar, M. A. Othman, M. F. A. Malek, and M. S. Zulkefli, "Impedance modeling for a unit cell of the square loop frequency selective surface at $2.4 \mathrm{GHz}$," 2013 Loughborough Antennas \& Propagation Conference (LAPC), 2013. http://dx.doi.org/10.1109/RFID-TA.2013.6694526.

[27] Chip Inductors - 0805HT. (n.d.). Coilcraft. [Online]. Available: http://www.coilcraft.com/pdfs/0805ht.pdf. Accessed Mar. 3, 2017.

[28] S. Tretyakov, Analytical modeling in applied electromagnetics. Boston: Artech House, 2003.

[29] C. L. Holloway and E. F. Kuester, "Net and partial inductance of a microstrip ground plane," IEEE Transactions on Electromagnetic Compatibility, vol. 40, no. 1, pp. 33-46, 1998. http://dx.doi.org/10.1109/15.659518.

[30] S.A. Tretyakov and C.R. Simovski, "Dynamic model of artificial reactive impedance surfaces," Journal of Electromagnetic waves and applications, vol. 17, iss. 1, 2003. http://dx.doi.org/10.1163/156939303766975407. 